\title{
Experimental observation of human bone marrow mesenchymal stem cell transplantation into rabbit intervertebral discs
}

\author{
HAO TAO ${ }^{1}$, YAZHOU LIN ${ }^{1}$, GUOQING ZHANG ${ }^{1}$, RUI GU $^{2}$ and BOHUA CHEN ${ }^{1}$ \\ ${ }^{1}$ Department of Orthopedic Surgery, The Affiliated Hospital of Qingdao University, \\ Qingdao, Shandong 266003; ${ }^{2}$ Department of Spinal and Neural Function Reconstruction, \\ China Rehabilitation Research Center, Beijing 100068, P.R. China
}

Received April 5, 2016; Accepted July 13, 2016

DOI: $10.3892 /$ br.2016.731

\begin{abstract}
Allogeneic bone marrow mesenchymal stem cell (BMSC) transplantation has been investigated worldwide. However, few reports have addressed the survival status of human BMSCs in the intervertebral discs (IVDs) in vivo following transplantation. The current study aimed to observe the survival status of human BMSCs in rabbit IVDs. The IVDs of 15 New Zealand white rabbits were divided into three groups: Punctured blank control group (L1-2); punctured physiological saline control group (L2-3); and punctured human BMSCs transfected with green fluorescent protein (GFP) group (L3-4, L4-5 and L5-6). One, 2, 4, 6 and 8 weeks after transplantation the IVDs were removed and a fluorescence microscope was used to observe the density of GFP-positive human BMSCs. The results indicated that in the sections of specimens removed at 1,2, 4, 6 and 8 weeks post-transplantation, no GFP-positive cells were observed in the control groups, whereas GFP-positive cells were apparent in the nucleus pulposus at all periods in the GFP-labeled human BMSCs group, and the cell density at 6 and 8 weeks was significantly less than that at 1,2 and 4 weeks post-transplantation $(\mathrm{P}<0.001)$. Thus, it was identified that human BMSCs were able to survive in the rabbit IVDs for 8 weeks.
\end{abstract}

\section{Introduction}

Approximately $80 \%$ of the aging population suffers from lower back pain (LBP) in their lifetime (1-3). It markedly affects the quality of life and work productivity, and significantly impacts health care spending (4). Intervertebral disc degeneration (IDD) is a common degenerative physiological process with

Correspondence to: Dr Bohua Chen, Department of Orthopedic Surgery, The Affiliated Hospital of Qingdao University, 59 Haier Road, Qingdao, Shandong 266003, P.R. China

E-mail: bhchen@hotmail.com

Key words: bone marrow mesenchymal stem cell, intervertebral disc, allogenic transplantation, animal experiment normal aging, which has been established as a causal factor with LBP $(5,6)$. Degenerative disorders of intervertebral discs (IVDs) are generally characterized by disequilibrium between extracellular matrix repair and degradative processes, including morphological and cellular changes (7-11).

A solution to managing disc degeneration would be to repair the IVDs, producing a matrix with similar or improved biological and biomechanical properties compared with the original (12). With this aim, cell transplantation has been widely investigated as an effective approach (12-14), including bone marrow mesenchymal stem cell (BMSC) transplantation. Using this method, BMSCs are transplanted into IVDs to replenish the matrix-producing cells, compensating for the decrease in matrix components. Thus far, the majority of studies focus on allogeneic BMSC transplantation in IVDs to observe the regeneration effect (15-18). However, there have been few reports regarding the survival status of human BMSCs in the IVDs post-transplantation in vivo. The current study was designed to observe the survival status of human BMSCs following transplantation into rabbit IVDs, providing an experimental basis for further investigation of disc degeneration treatment.

\section{Materials and methods}

Animals and BMSCs. Fifteen New Zealand white rabbits [Animal Husbandry and Veterinary Bureau, Shandong Academy of Agricultural sciences; permission no. SCXK (Lu) 20040013], aged 6-months and of either gender (weight, 2.0-3.0 kg) were used in the study. The rabbits were maintained apart at room temperature with 16:8 h light and dark cycles, and were fed with rabbit feed (Qingdao Kangda Foodstuffs Co., Ltd., Qingdao, China). All animals were raised, and procedures were performed, in strict accordance with Animal Ethical Standards, and the study was approved by the Ethics Committee of the Affiliated Hospital of Qingdao University (Qingdao, China). The IVDs of these rabbits were divided into three groups: Punctured blank control group (L1-2), punctured physiological saline (PS) control group (L2-3) and punctured human BMSCs transfected with green fluorescent protein (GFP) group (L3-4, L4-5 and L5-6).

GFP-labeled human BMSCs and the complete medium [Dulbecco's modified Eagle's medium with $15 \%$ fetal bovine 

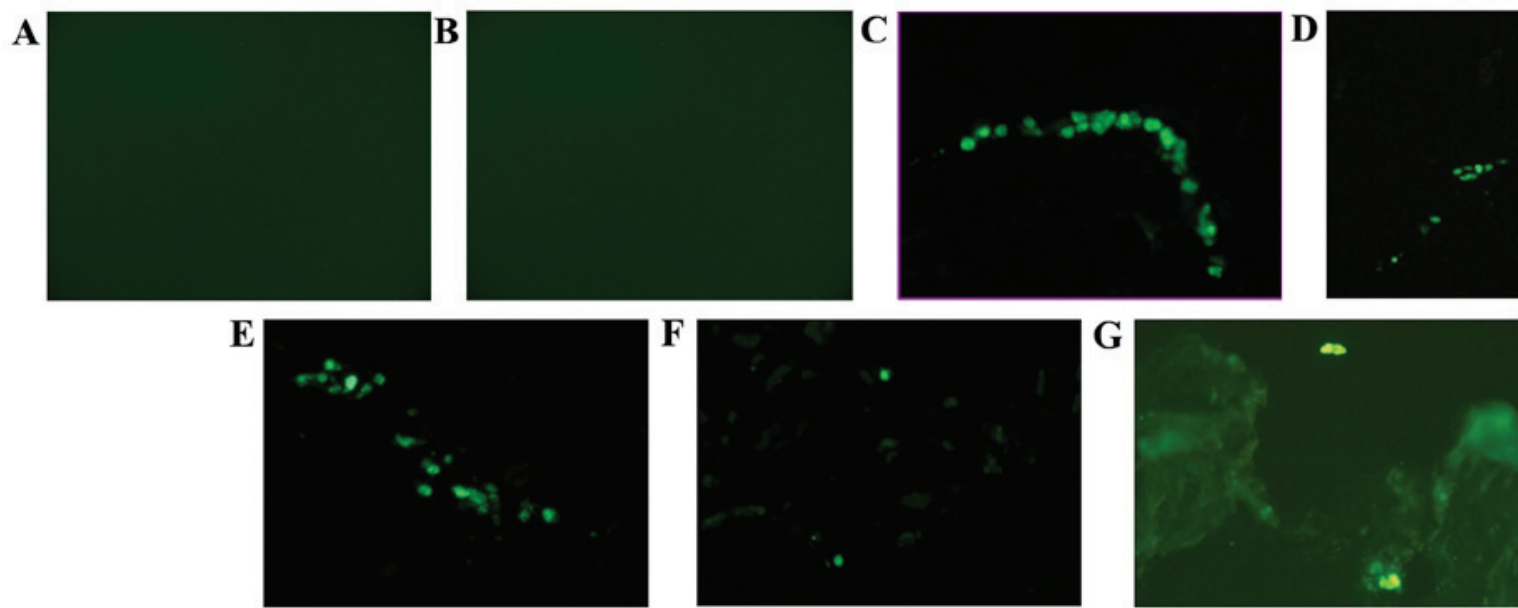

Figure 1. Microscopic image of fluorescent cells at different time-points post-bone marrow mesenchymal stem cell transplantation (magnification, x200). No GFP-positive cells were detected in the (A) punctured blank control group (L1-2) and (B) punctured physiological saline control group (L2-3). GFP-positive cells were observed in the nucleus pulposus at (C) 1 week, (D) 2 weeks, (E) 4 weeks, (F) 6 weeks and (G) 8 weeks post-transplantation of GFP-labeled human bone marrow mesenchymal stem cells. GFP, green fluorescent protein.

serum] were purchased from Cyagen Bioscience Co., Ltd. (Santa Clara, CA, USA). The BMSCs were frozen prior to use.

Preparation of GFP-labeled human BMSC suspension. Frozen GFP-labeled human BMSCs were thawed and cultured in a $25 \mathrm{~cm}^{2}$ culture flask with complete medium. After overspreading the bottom of the flask, cells were trypsinized, centrifuged at $520 \mathrm{x}$ g for $5 \mathrm{~min}$ at room temperature and suspended in normal saline at a final concentration of $1 \times 10^{6}$ cells $/ \mathrm{ml}$ and prepared for transplantation.

Transplantation of GFP-labeled human BMSCs. After tranquilizing the rabbits by intramuscular injection of ketamine hydrochloride (40 mg/kg; Selleck Chemicals, Houston, TX, USA) and xylazine (2.5 mg/kg; Selleck Chemicals), $25 \mu 1$ GFP-labeled human BMSC suspension was injected into the L3-L4, L4-L5, L5-L6 discs of the rabbits using a posterolateral approach with a 29-gauge needle on a $100 \mu 1$ microinjector. The same method was used to inject the equivalent PS into the L2-3 discs. Following the surgical procedure, 20 units benzylpenicillin sodium and $0.25 \mathrm{mg}$ streptomycin were administered by injection to prevent infection.

Evaluation of the survival of BMSCs. The rabbits were sacrificed by ear vein air injection at 1, 2, 4, 6 and 8 weeks post-treatment ( $n=3$ per time-point). Their spines were harvested and the discs were isolated. The specimens were frozen in liquid nitrogen immediately after harvest and cryosectioned axially at $6 \mu \mathrm{m}$. A fluorescence microscope was used to observe the cell density in the different groups. To compare the ralative cell density, cells in five sections crossing the center of the discs per group were counted using x200 magnification images.

Statistical analysis. Statistical analysis was performed with SPSS 16.0 (SPSS, Inc., Chicago, IL, USA). A signed-rank test was used to analyze the data and $\mathrm{P}<0.05$ was considered to indicate a statistically significant difference.

\section{Results}

General status of laboratory animals. Prior to transplantation, two rabbits succumbed due to environment inadaptation and were substituted with two different rabbits.

There were no complications or mortalities during and after transplantation. After the specimens were harvested, no significant changes were observed macroscopically and, microscopically, no vascularization or inflammation was apparent in the nucleus pulposus (NP).

Survival status of human BMSCs at different periods post-transplantation. No GFP-positive cells were detected in the blank and PS control groups, whereas GFP-positive cells were observed in the NP at all periods post-transplantation in the BMSCs group, demonstrating the survival of the transplanted BMSCs (Fig. 1). In the BMSCs group, a mean of 11, 10, 11, 2 and 1 GFP-positive cells was detected in one section at 1, 2, 4, 6 and 8 weeks post-transplantation. Significant differences were identified between the cell densities at different periods $(\mathrm{H}=28.27 ; \mathrm{P}<0.001)$. The cell density at 6 and 8 weeks was significantly less than that at 1,2 and 4 weeks post-transplantation $(\mathrm{H}=16.50-24.44 ; \mathrm{P}<0.001)$ and no statistical differences were observed between the cell density counted at 1, 2, 4, 6 and 8 weeks post-transplantation $(\mathrm{H}=2.06-5.44 ; \mathrm{P}>0.05)$.

\section{Discussion}

More than $20 \%$ of all LBP originates from degenerative changes in IVDs, leading to a gradual process of cellular loss and degradation of the extracellular matrix within the IVDs (19). While the pathology of IDD is not fully understood, insufficient nutritional supply to the disc has been proposed as a possible initiator (20). As the largest avascular organs in humans, IVDs obtain nutrition from adjacent vertebral bodies by diffusion through the annulus fibrosus (AF) and endplate. Among these nutritional routes, the endplate pathway has been identified to be more significant than the other AF signaling pathways (21). In cases with loss of endplate permeability 
and/or disruptions therein, as well as changes in geometry and fall in diffusivity associated with fluid expression, the nutrient concentrations could fall to levels inadequate to maintain cellular activity or viability, thus initiating or accelerating disc degeneration (22).

Current treatments for degenerative disc disease including conservative treatment (medications and physiotherapy) and surgical treatment (spinal fusion or total disc replacement) may alleviate pain, but fail to address the underlying issue and often result in reoccurrence in the same or adjacent discs (23). Particularly in treatment with spinal fusion, it accelerates the degeneration of adjacent IVDs and the severity of adjacent segment degeneration increases with the fused segment numbers (24). Cell-based tissue engineering, which aims to restore IVD structure and function, represents a promising approach for IVD repair or regeneration.

Since NP cells possess a chondrocyte-like phenotype (25), MSCs, which were capable of differentiating into a chondrocyte-like phenotype following appropriate stimulation $(12,26)$, have shown promise as suitable seed cells to be widely applied for IVD regeneration (27-29). In addition, MSCs have the advantage of easy availability and proliferative ability, better potential in producing matrix (mainly comprising type II collagen and proteoglycan) and low immunogenicity (29-32). BMSCs, as the most focused seed cells, have been demonstrated as effective in decelerating disc degeneration in experimental models by autologous transplantation (29) and allogeneic BMSC transplantation has also become a focus of investigation worldwide (15-18).

Among research investigating allogeneic BMSC transplantation, there are few studies regarding human BMSCs. In the current study, a GFP gene was used as a marker to observe the survival status of human BMSCs in rabbit IVDs following transplantation. The results indicate that human BMSCs may survive in rabbits for 8 weeks, but the cell density decreased significantly from 6 to 8 weeks. Rather than applying scaffolds, direct injection avoids the underlying influence on the microenvironment within IVDs. Furthermore, no vascularization and inflammation was identified in the NP, which implies little interference of surgical procedures and low immunogenicity of human BMSCs. In the GFP-labeled human BMSCs group, the density of GFP-positive cells marginally decreased at 2 weeks and significantly decreased after 6 weeks post-transplantation, which may be due to various reasons, including inadaptation to the IVD microenvironment, insufficient nutrients for cell survival and cell migration to the outer NP.

In conclusion, the present study observed the survival status of human BMSCs in rabbit IVDs and demonstrated that the cells were able to survive during 8 weeks of transplantation. The finding provides fundamental information for tissue engineering repair used to decelerate the degeneration of discs. The status of the cells 8 weeks post-transplantation, such as their ability to survive, proliferate and differentiate, requires further investigation.

\section{Acknowledgements}

The authors would like to thank the members for their enthusiastic participation in the present study. The study was supported by a research grant award from the National Natural Science Foundation of China (grant no. 81371998) and the National Key Clinical Specialty construction projects of China (grant no. 201030402).

\section{References}

1. Hoy D, Brooks P, Blyth F and Buchbinder R: The Epidemiology of low back pain. Best Pract Res Clin Rheumatol 24: 769-781, 2010.

2. Takahashi K, Aoki Y and Ohtori S: Resolving discogenic pain. Eur Spine J 17 (Suppl 4): 428-431, 2008.

3. Hillman M, Wright A, Rajaratnam G, Tennant A and Chamberlain MA: Prevalence of low back pain in the community: Implications for service provision in Bradford, UK. J Epidemiol Community Health 50: 347-352, 1996.

4. McMeeken J, Tully E, Stillman B, Nattrass C, Bygott IL and Story I: The experience of back pain in young Australians. Man Ther 6: 213-220, 2001

5. Pye SR, Reid DM, Smith R, Adams JE, Nelson K, Silman AJ and O'Neill TW: Radiographic features of lumbar disc degeneration and self-reported back pain. J Rheumatol 31: 753-758, 2004.

6. Hoyland JA, Le Maitre C and Freemont AJ: Investigation of the role of IL-1 and TNF in matrix degradation in the intervertebral disc. Rheumatology (Oxford) 47: 809-814, 2008.

7. Le Maitre CL, Freemont AJ and Hoyland JA: Localization of degradative enzymes and their inhibitors in the degenerate human intervertebral disc. J Pathol 204: 47-54, 2004.

8. Le Maitre CL, Freemont AJ and Hoyland JA: Accelerated cellular senescence in degenerate intervertebral discs: A possible role in the pathogenesis of intervertebral disc degeneration. Arthritis Res Ther 9: R45, 2007.

9. Richardson SM, Knowles R, Tyler J, Mobasheri A and Hoyland JA: Expression of glucose transporters GLUT-1, GLUT-3, GLUT-9 and HIF-1alpha in normal and degenerate human intervertebral disc. Histochem Cell Biol 129: 503-511, 2008.

10. Zhao CQ, Wang LM, Jiang LS and Dai LY: The cell biology of intervertebral disc aging and degeneration. Ageing Res Rev 6: 247-261, 2007.

11. Kim KW, Chung HN, Ha KY, Lee JS and Kim YY: Senescence mechanisms of nucleus pulposus chondrocytes in human intervertebral discs. Spine J 9: 658-666, 2009.

12. Richardson SM, Walker RV, Parker S, Rhodes NP, Hunt JA, Freemont AJ and Hoyland JA: Intervertebral disc cell-mediated mesenchymal stem cell differentiation. Stem Cells 24: 707-716, 2006.

13. Sobajima S, Vadala G, Shimer A, Kim JS, Gilbertson LG and Kang JD: Feasibility of a stem cell therapy for intervertebral disc degeneration. Spine J 8: 888-896, 2008.

14. Sakai D, Mochida J, Iwashina T, Watanabe T, Nakai T, Ando K and Hotta T: Differentiation of mesenchymal stem cells transplanted to a rabbit degenerative disc model: Potential and limitations for stem cell therapy in disc regeneration. Spine 30: 2379-2387, 2005

15. Wang YH, Yang B, Li WL and Li JM: Effect of the mixture of bone marrow mesenchymal stromal cells and annulus fibrosus cells in repairing the degenerative discs of rabbits. Genet Mol Res 14: 2365-2373, 2015.

16. Yi Z, Guanjun T, Lin C and Zifeng P: Effects of transplantation of htimp1-expressing bone marrow mesenchymal stem cells on the extracellular matrix of degenerative intervertebral discs in an in vivo rabbit model. Spine 39: E669-E675, 2014.

17. Wang Y, Wu X, Wang F, Hong X, Bao J and Zhu L: An in vitro study on biological characteristics of bone marrow mesenchymal stem cells in microenvironment of premature senescence of nucleus pulposus cells. Zhongguo Xiu Fu Chong Jian Wai Ke Za Zhi 28: 758-762, 2014 (In Chinese).

18. Subhan RA, Puvanan K, Murali MR, Raghavendran HR, Shani S, Abdullah BJ, Abbas AA, Mohamed JA and Kamarul T: Fluoroscopy assisted minimally invasive transplantation of allogenic mesenchymal stromal cells embedded in HyStem reduces the progression of nucleus pulposus degeneration in the damaged ntervertebral disc: A preliminary study in rabbits. ScientificWorldJournal 2014: 818502, 2014.

19. Dagenais S, Caro J and Haldeman S: A systematic review of low back pain cost of illness studies in the United States and internationally. Spine J 8: 8-20, 2008.

20. Grunhagen T, Shirazi-Adl A, Fairbank JC and Urban JP: Intervertebral disk nutrition: A review of factors influencing concentrations of nutrients and metabolites. Orthop Clin North Am 42: 465-477, vii, 2011. 
21. Ogata K and Whiteside LA: 1980 Volvo award winner in basic science. Nutritional pathways of the intervertebral disc. An experimental study using hydrogen washout technique. Spine 6: 211-216, 1981.

22. Soukane DM, Shirazi-Adl A and Urban JP: Computation of coupled diffusion of oxygen, glucose and lactic acid in an intervertebral disc. J Biomech 40: 2645-2654, 2007.

23. Richardson SM, Mobasheri A, Freemont AJ and Hoyland JA: Intervertebral disc biology, degeneration and novel tissue engineering and regenerative medicine therapies. Histol Histopathol 22: 1033-1041, 2007.

24. Levin DA, Hale JJ and Bendo JA: Adjacent segment degeneration following spinal fusion for degenerative disc disease. Bull NYU Hosp Jt Dis 65: 29-36, 2007.

25. Sive JI, Baird P, Jeziorsk M, Watkins A, Hoyland JA and Freemont AJ: Expression of chondrocyte markers by cells of normal and degenerate intervertebral discs. Mol Pathol 55: 91-97, 2002.

26. Steck E, Bertram H, Abel R, Chen B, Winter A and Richter W: Induction of intervertebral disc-like cells from adult mesenchymal stem cells. Stem Cells 23: 403-411, 2005.

27. Hiyama A, Mochida J, Iwashina T, Omi H, Watanabe T, Serigano K, Tamura F and Sakai D: Transplantation of mesenchymal stem cells in a canine disc degeneration model. J Orthop Res 26: 589-600, 2008.
28. Sakai D, Mochida J, Iwashina T, Hiyama A, Omi H, Imai M, Nakai T, Ando K and Hotta T: Regenerative effects of transplanting mesenchymal stem cells embedded in atelocollagen to the degenerated intervertebral disc. Biomaterials 27: 335-345, 2006.

29. Sakai D, Mochida J, Yamamoto Y, Nomura T, Okuma M, Nishimura K, Nakai T, Ando K and Hotta T: Transplantation of mesenchymal stem cells embedded in Atelocollagen gel to the intervertebral disc: A potential therapeutic model for disc degeneration. Biomaterials 24: 3531-3541, 2003.

30. Bartholomew A, Sturgeon C, Siatskas M, Ferrer K, McIntosh K, Patil S, Hardy W, Devine S, Ucker D, Deans R, et al: Mesenchymal stem cells suppress lymphocyte proliferation in vitro and prolong skin graft survival in vivo. Exp Hematol 30: 42-48, 2002.

31. Horwitz EM, Prockop DJ, Fitzpatrick LA, Koo WW, Gordon PL, Neel M, Sussman M, Orchard P, Marx JC, Pyeritz RE, et al: Transplantability and therapeutic effects of bone marrow-derived mesenchymal cells in children with osteogenesis imperfecta. Nat Med 5: 309-313, 1999.

32. Le Blanc K, Tammik C, Rosendahl K, Zetterberg E and Ringdén O: HLA expression and immunologic properties of differentiated and undifferentiated mesenchymal stem cells. Exp Hematol 31: 890-896, 2003. 